\title{
Percepción en la implementación de modelos de gestión educativa en instituciones de educación superior
}

\author{
Perception in the implementation of educational management models in higher education \\ institutions
}

\author{
Edgar Olguín Guzmán a, Ana Lucía Fallas Garro b
}

\begin{abstract}
:
This article shows the results in the perception of students enrolled in three institutions of higher education, during the stage of implementation of an educational management model that allows to favor the institutional operation, specifically in the processes of admission, permanence, graduation and qualification.

Through this document, it is intended to publicize the way in which methods and procedures were applied according to the problems presented in educational institutions, in educational management processes, in order to obtain benefits in their application environment .

The lack of follow-up of educational institutions, as well as the presence of students with an income profile that is far from the required one, have led students to develop with poor educational management processes, which leads to innovating in their nature with the purpose of strengthening its application.

For this, an non-experimental, quantitative, transversal, descriptive and correlational methodology was used, which is supported by a comparative analysis of good practices in higher education institutions, whose results serve as a prototype to follow to generate a comprehensive institutional educational management model, which strengthens the daily operation of the institutions that adopt it.

The results obtained in the implementation of the elements that make up the integral institutional model of educational management for higher education institutions are presented, where the main actors of the teaching and learning process participated: managers, teachers and students.
\end{abstract}

Keywords:

Educational Management, Strategic Management, Management Models, Comparative Analysis, Educational Projects.

\section{Resumen:}

El presente artículo muestra los resultados en la percepción de los alumnos matriculados en tres instituciones de educación superior, durante la etapa de implementación de un modelo de gestión educativa que permita favorecer la operación institucional, específicamente en los procesos de ingreso, permanencia, egreso y titulación.

A través del presente documento se pretende dar a conocer la forma en la cual se aplicaron métodos y procedimientos acordes a las problemáticas que se presentan en las instituciones educativas, en los procesos de gestión educativa, con la finalidad de obtener beneficios en su entorno de aplicación.

\footnotetext{
a Profesor Investigador. Universidad Autónoma del Estado de Hidalgo. Instituto de Ciencias Básicas e Ingeniería. https://orcid.org/00000002-9003-6511. eolguin@uaeh.edu.mx. Doctorando de la Fundación Universitaria Iberoamericana FUNIBER. Universidad Internacional Iberoamericana UNINI, México.

b Doctora en Educación con énfasis en Administración Educativa, colaboradora del CTP de Acosta del Ministerio de Educación Pública, Costa Rica. Directora de Tesis del Doctorado en Proyectos. Fundación Universitaria Iberoamericana FUNIBER. Universidad Internacional
} 
La falta de seguimiento de las instituciones educativas, así como, la presencia de alumnos con perfil de ingreso alejado del requerido, han propiciado que los alumnos se desarrollen con procesos de gestión educativa deficientes, lo que conlleva a inn ovar en la naturaleza de ellos con la finalidad de fortalecer su aplicación.

Para ello se utilizó una metodología de tipo no experimental, cuantitativo, transversal, descriptivo y correlacional, que se sustenta por un análisis comparativo de buenas prácticas en instituciones de educación superior, cuyos resultados sirven como prototipo a seguir para generar un modelo de gestión educativa institucional integral, que fortalezca la operación cotidiana de las instituciones que lo adopten.

Se presentan los resultados obtenidos en la implementación de los elementos que conforman el Modelo Institucional Integral de gestión educativa, para instituciones de educación superior, en donde participaron los principales actores del proceso de ens eñanza y aprendizaje: directivos, docentes y alumnos.

\section{Palabras Clave:}

Gestión Educativa, Gestión Estratégica, Modelos de Gestión, Análisis Comparativo, Proyectos Educativos.

\section{Introducción}

Actualmente, el campo de la gestión educativa, representa un campo de investigación muy importante a favor del fortalecimiento de las instituciones educativas en todos los niveles, en el nivel superior, representa una oportunidad de consolidación de los procesos administrativos y de seguimiento de los alumnos matriculados en los diferentes programas educativos.

El presente artículo de investigación plantea la necesidad del diseño de un modelo de gestión educativa, que sea adaptable a las necesidades vigentes, de cada uno de los elementos que conforman la estructura organizacional de una institución educativa de nivel superior.

Para lo anterior, se plantea un análisis previo de instituciones de educación superior, que permita tener un referente acerca de los procesos administrativos y de gestión, con la finalidad de detectar buenas prácticas que sirvan como punto de partida al momento de plantear un modelo de gestión educativa.

Para lograr consolidar la investigación con un sustento real, que sirva de referencia al momento de establecer modelos de gestión, se han involucrado a tres instituciones de educación superior.

Los resultados que se obtengan, servirán en gran medida, primero para conocer la situación actual de las instituciones inmersas en la investigación, en cuanto a las condiciones actuales que tienen de gestión educativa, y en segundo término, identificar las características del modelo de gestión educativa que se proponga, partiendo de las necesidades actuales y de las condiciones vigentes de los procesos de gestión educativa, en cuanto a procesos de ingreso, permanencia, egreso y titulación, en instituciones de educación superior.

La estructuración de la investigación que respalda este documento, se encuentra integrada por los conceptos básicos de la gestión de proyectos, lo anterior por tratarse de un proyecto con un sustento científico enfocado al ámbito educativo, por lo que resultó pertinente resaltar algunos conceptos básicos de la gestión de proyectos, iniciando desde el marco teórico, tipos de proyecto, características de ellos, entre otras generalidades.

Por otro lado, se abordaron algunas aportaciones teóricas relevantes que enmarcan y sustentan la conceptualización misma de la gestión educativa, con la finalidad de lograr una mancuerna interesante, entre proyectos y gestión educativa, que sirva como una sólida fundamentación teórica, que otorgue un sustento científico a la investigación.

\section{Metodología}

La metodología empleada en el desarrollo presente trabajo de investigación fue del tipo no experimental, cuantitativo, transversal, descriptivo y correlacional.

La población objeto de estudio estuvo conformada por los estudiantes inscritos, profesores en activo y funcionarios que desde su perspectiva, tienen contacto con los procesos de gestión educativa.

Se estructuró un análisis comparativo entre tres instituciones diferentes, considerando las opiniones de docentes, estudiantes y personal directivo, que de una u otra forma, tiene que ver con el proceso de toma de decisiones a nivel institucional.

Se utilizó un instrumento que consta de ítems articulados de tal forma, que permitan identificar el nivel de uso de cada modelo de gestión educativa, así se pudo obtener un análisis comparativo de su uso, en donde se retomaron los resultados de la encuesta en distintas perspectivas.

De acuerdo a las opiniones recuperadas, se generaron decisiones que están ligadas a la incorporación de un modelo institucional integral de gestión educativa, que sirva como marco de referencia en la funcionalidad, 
operación y dirección estratégica en instituciones con oferta educativa en niveles licenciatura y posgrado.

Se presentan en las figuras subsecuentes, las respuestas de los actores entrevistadas, que ofrecen un soporte y sustento ligado a las circunstancias vigentes de cada organización.

De las personas entrevistadas, el $69.2 \%$ corresponde al género femenino, y el $30.8 \%$, corresponde al género masculino, en los perfiles de estudiante, docentes y funcionarios.

Género

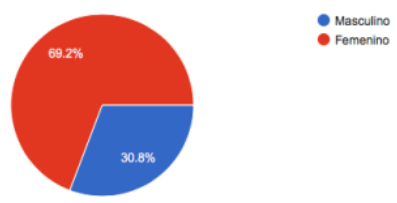

Gráfica No. 1: Distribución de usuarios por género que participaron en el instrumento de evaluación,

Fuente: Resultados obtenidos de encuesta en línea, elaboración propia.

Tipo de contratación en su institución.

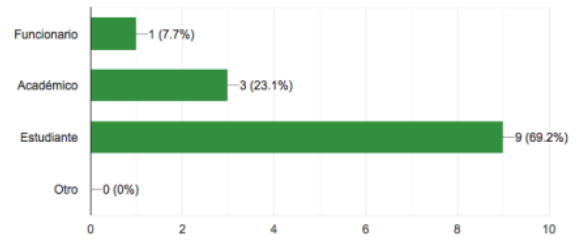

Gráfica No. 2: Distribución de usuarios por tipo de contratación que participaron en el instrumento de evaluación,

Fuente: Resultados obtenidos de encuesta en línea, elaboración propia.

Los tipos de contratación en los que se tuvo acercamiento fueron los funcionarios, administrativos, docentes y estudiantes.

De las personas entrevistadas, el $7.7 \%$ corresponde a funcionarios de su respectiva institución, el $23.1 \%$, corresponde a académicos, y el $69.2 \%$ a estudiantes.

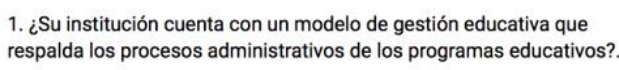

1. ¿Su institución cuenta con un modelo de gestión educativa que respalda los procesos administrativos de los programas educativos?

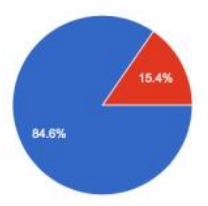

$\because$ si

Gráfica No. 3. Existencia de un modelo de gestión educativa que respalde los procesos administrativos de las instituciones que participaron en el instrumento de evaluación,

Fuente: Resultados obtenidos de encuesta en línea, elaboración propia

El $15.4 \%$ de las personas entrevistadas, indicaron que su institución no cuenta con un modelo de gestión educativa que respalde los procesos administrativos, mientras que el $84.6 \%$ indicó que si cuenta con modelo de gestión educativa que respalde los procesos administrativos.

3. ¿Existe seguimiento cercano a los procesos de ingreso, permanencia, egreso y titulación en sus programas educativos?.

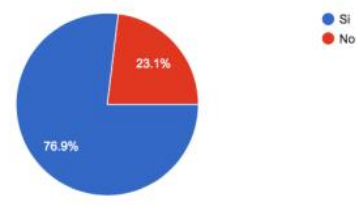

Gráfica No. 4. Seguimiento cercano a los procesos de ingreso, permanencia, egreso y titulación, de acuerdo al modelo de gestión educativa existente

Fuente: Resultados obtenidos de encuesta en línea, elaboración propia.

Las instituciones que participaron en el análisis comparativo de prácticas de gestión educativa fueron:

\begin{tabular}{|c|c|c|}
\hline $\begin{array}{c}\text { Nombre de la } \\
\text { Institución } \\
\text { Educativa }\end{array}$ & $\begin{array}{c}\text { No. de } \\
\text { participantes } \\
\text { en el } \\
\text { instrumento } \\
\text { de análisis. }\end{array}$ & $\begin{array}{c}\text { Nivel de } \\
\text { estudios de } \\
\text { los } \\
\text { participantes. }\end{array}$ \\
\hline $\begin{array}{c}\text { Universidad } \\
\text { Autónoma del } \\
\text { Estado de } \\
\text { Hidalgo. }\end{array}$ & 10 & Maestría \\
\hline
\end{tabular}




\begin{tabular}{|c|c|c|}
\hline Centro & 10 & $\begin{array}{c}\text { Licenciatura y } \\
\text { Maestría } \\
\text { Cultural de } \\
\text { Estudios } \\
\text { Superiores. }\end{array}$ \\
\hline $\begin{array}{c}\text { Universidad } \\
\text { Nacional } \\
\text { Autónoma de } \\
\text { México. }\end{array}$ & 10 & Doctorado \\
\hline
\end{tabular}

Tabla No. 1: Instituciones que participaron en el análisis comparativo de prácticas de gestión educativa. Elaboración propia (2019).

\section{Resultados}

A través de los resultados obtenidos en el estudio comparativo de buenas prácticas en cuanto a gestión educativa en las diversas instituciones que participaron, se logró proponer un modelo de gestión educativa que resultara integral y transversal a las instituciones educativas que ofrezcan educación superior.

El modelo resultante es llamado Modelo Institucional Integral de Gestión Educativa (MIIGE) y se estructura por las siguientes dimensiones:

- Estratégica

- Académica

- Tecnológica

- Normativa

- Operativa

- Institucional

Estratégica. Como parte básica se propone la implementación de una dimensión cuya finalidad sea el permitir contar con un apartado en donde se puedan integrar aquellos elementos relacionados a la parte estratégica de las instituciones educativas que adopten el modelo en su quehacer diario, como parte de la integración de mecanismos de instrumentación para guiar cada uno de los procesos básicos de gestión estratégica.

\begin{tabular}{|l|c|}
\hline Elemento & Descripción \\
\hline Visión de largo plazo & $\begin{array}{c}\text { Con una visión } \\
\text { definida para un } \\
\text { tiempo de diez años. }\end{array}$ \\
\hline Liderazgo & $\begin{array}{c}\text { Mostrado en todas las } \\
\text { áreas que conformen } \\
\text { la institución, } \\
\text { incluyendo áreas }\end{array}$ \\
\hline
\end{tabular}

\begin{tabular}{|c|c|}
\hline & operativas \\
\hline Dirección estratégica & $\begin{array}{c}\text { Con la presencia de un } \\
\text { Director con } \\
\text { experiencia suficiente } \\
\text { en gestión de } \\
\text { instituciones } \\
\text { educativas }\end{array}$ \\
\hline $\begin{array}{l}\text { Gestión de recursos } \\
\text { humanos }\end{array}$ & $\begin{array}{c}\text { Esta competencia será } \\
\text { requerida de forma } \\
\text { transversal y no } \\
\text { vertical, con la } \\
\text { finalidad de establecer } \\
\text { parámetros de } \\
\text { participación por parte } \\
\text { de los colaboradores } \\
\text { de las instituciones } \\
\text { educativas, donde la } \\
\text { comunicación sea un } \\
\text { proceso permanente. }\end{array}$ \\
\hline Planeación Estratégica & $\begin{array}{c}\text { Implementación de } \\
\text { procesos de } \\
\text { planeación estratégica, } \\
\text { donde se tenga } \\
\text { claridad de las metas } \\
\text { de corto, mediano y } \\
\text { largo plazo. }\end{array}$ \\
\hline Definición de Objetivos & $\begin{array}{c}\text { Establecer objetivos } \\
\text { organizacionales con } \\
\text { visión de largo plazo, } \\
\text { en donde se } \\
\text { especifiquen de } \\
\text { manera concreta las } \\
\text { acciones a seguir y los } \\
\text { productos a obtener }\end{array}$ \\
\hline $\begin{array}{l}\text { Seguimiento de } \\
\text { acciones }\end{array}$ & $\begin{array}{c}\text { Definición y } \\
\text { seguimiento de } \\
\text { acciones en donde se } \\
\text { tenga claridad de los } \\
\text { recursos y actividades }\end{array}$ \\
\hline
\end{tabular}




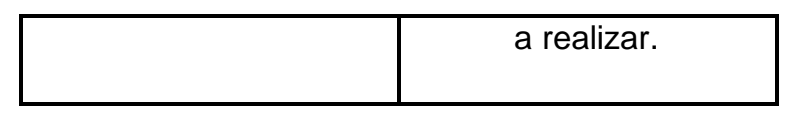

Tabla No. 2: Elementos de la Dimensión Estratégica del Modelo Institucional Integral de Gestión Educativa Propuesto.

Elaboración propia (2019).

Logrando la integración de los elementos anteriores, se obtiene una forma de identificar el crecimiento institucional a través de mecanismos de seguimiento dispuestos a generar un cambio sustancial en las áreas que formen parte de la institución.

Se debe recordar que una institución bien dirigida, tendrá un impacto en todos los niveles de su estructura organizacional, logrando resultados que se verán reflejados en los egresados de sus programas educativos.

Académica. En las instituciones de educación superior, es muy importante separar el componente académico del componente administrativo, por lo que en el presente modelo de gestión educativa que se propone, se requiere especificar una dimensión que esté relacionada al componente académico de las instituciones educativas.

Si bien es cierto, el elemento académico es uno de los más importantes para el crecimiento de las organizaciones de educación superior, se debe tener mucho cuidado al momento de establecer los componentes que de manera integral, formarán parte de la dimensión académica, además servirá para manifestar cada una de las necesidades que en este ámbito tengan los colaboradores de las instituciones educativas de nivel superior.

\begin{tabular}{|c|c|}
\hline Elemento & Descripción \\
\hline $\begin{array}{c}\text { Evaluación } \\
\text { programanente de } \\
\text { de nivel superior }\end{array}$ & $\begin{array}{c}\text { Se deberá seguir un } \\
\text { plan de evaluación de } \\
\text { programas educativos, } \\
\text { para ello se deberá } \\
\text { revisar los organismos } \\
\text { existentes. Dicho plan } \\
\text { deberá ser más } \\
\text { frecuente que los que } \\
\text { ofrezcan los } \\
\text { organismos externos. }\end{array}$ \\
\hline $\begin{array}{c}\text { Cuidado de la calidad } \\
\text { educativa en cada uno } \\
\text { de los grupos } \\
\text { asignados a cada } \\
\text { programa educativo }\end{array}$ & $\begin{array}{c}\text { Implementar } \\
\text { estrategias de } \\
\text { seguimiento en cada } \\
\text { una de las sesiones } \\
\text { académicas que se }\end{array}$ \\
\hline
\end{tabular}

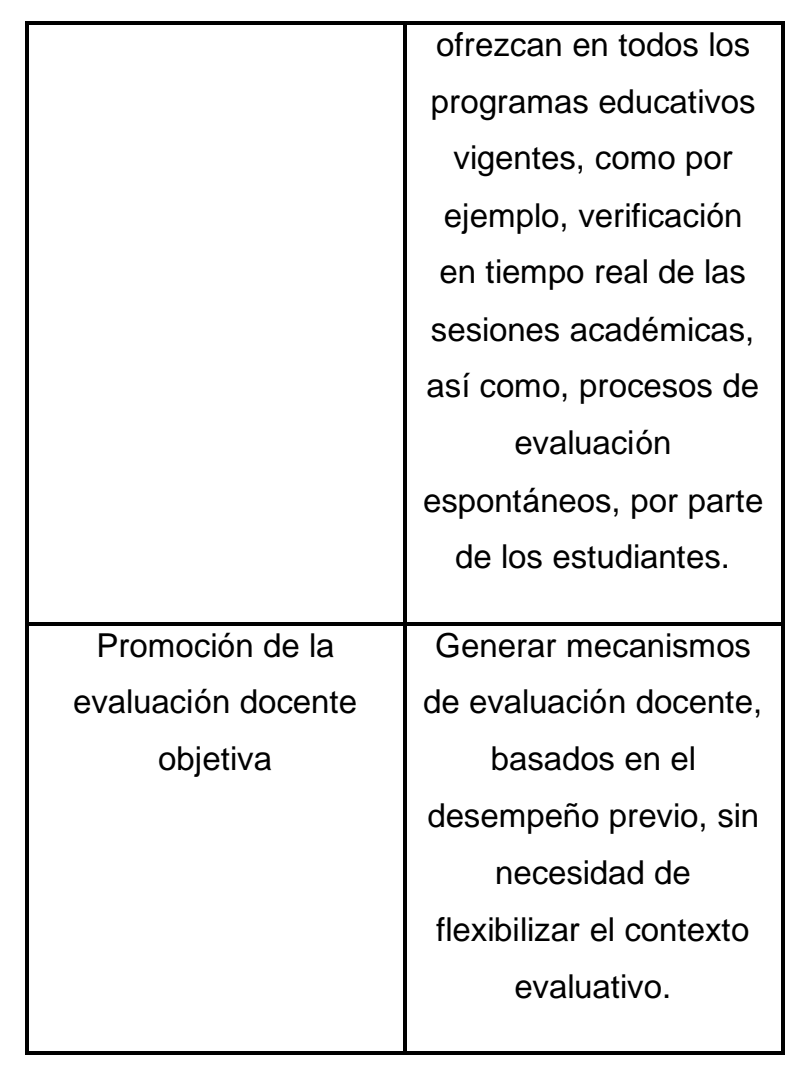

Tabla No. 3: Elementos de la Dimensión Académica del Modelo Institucional Integral de Gestión Educativa Propuesto.

Elaboración propia (2019).

Una vez que la dimensión académica se encuentre vigente, es posible iniciar con un nivel de mejora que impacte en los índices de ingreso, retención, egreso y titulación.

Tecnológica. Como parte fundamental del modelo de gestión educativa que se propone, se encuentra la Dimensión Tecnológica, cuya finalidad será establecer la estructura tecnológica que estará soportando cada una de las funciones, procedimientos y procesos tecnológicos relacionados con la operación diría y recurrente de la institución educativa.

Este componente muy necesario del modelo de gestión educativa, de ninguna manera debe conceptualizarse como únicamente la infraestructura tecnológica y el uso de herramientas de vanguardia, debe considerarse como una cultura, cuya base fundamental será el uso de la tecnología como recurso para la gestión, es decir, se debe considerar a la tecnología como el medio y no como el fin, el medio para lograr procesos de gestión educativa ligeros y que se tengan en un contexto de uso común, que permitan el fortalecimiento de la parte operativa en cada uno de los procesos internos que se tengan en la institución educativa que adopte el modelo. 


\begin{tabular}{|c|c|}
\hline Elemento & Descripción \\
\hline $\begin{array}{l}\text { Infraestructura basada } \\
\text { en sistemas de } \\
\text { telecomunicaciones. }\end{array}$ & $\begin{array}{l}\text { Conexión a internet, } \\
\text { uso de servicios de la } \\
\text { red interna corporativa } \\
\text { (intranet). }\end{array}$ \\
\hline $\begin{array}{l}\text { Dispositivos portátiles } \\
\text { para cada colaborador } \\
\text { que forma parte de la } \\
\text { institución educativa. }\end{array}$ & $\begin{array}{l}\text { Cada colaborador de } \\
\text { la institución } \\
\text { educativa, tendrá a su } \\
\text { resguardo para su uso } \\
\text { institucional, un } \\
\text { dispositivo portátil. }\end{array}$ \\
\hline $\begin{array}{l}\text { Uso de plataforma } \\
\text { educativa como parte } \\
\text { de una estrategia } \\
\text { transversal a nivel } \\
\text { institucional. }\end{array}$ & $\begin{array}{l}\text { Se promoverá el uso } \\
\text { de la plataforma } \\
\text { educativa, como una } \\
\text { forma de mejorar el } \\
\text { aprovechamiento de } \\
\text { los recursos } \\
\text { tecnológicos. }\end{array}$ \\
\hline $\begin{array}{l}\text { Desarrollo de sistemas } \\
\text { de información a la } \\
\text { medida de las } \\
\text { problemáticas de la } \\
\text { institución educativa. }\end{array}$ & $\begin{array}{l}\text { Se prevé la } \\
\text { intervención de } \\
\text { personal experto en } \\
\text { materia de desarrollo } \\
\text { de sistemas, ya sea } \\
\text { personal que forme } \\
\text { parte de la institución, } \\
\text { o bien, la contratación } \\
\text { de outsourcing. }\end{array}$ \\
\hline $\begin{array}{l}\text { Soluciones integradas } \\
\text { y desarrolladas a partir } \\
\text { de estrategias de } \\
\text { información } \\
\text { almacenadas en la } \\
\text { nube. }\end{array}$ & $\begin{array}{l}\text { Se } r \text { estará } \\
\text { promoviendo de } \\
\text { proceso un } \\
\text { capacitación } \\
\text { permanente a los } \\
\text { colaboradores de la } \\
\text { institución educativa, } \\
\text { en el uso de } \\
\text { aplicaciones en la } \\
\text { nube para almacenar } \\
\text { grandes cantidades de } \\
\text { información, resultado } \\
\text { de los procesos } \\
\text { operativos diarios. }\end{array}$ \\
\hline
\end{tabular}

Tabla No. 4: Elementos de la Dimensión Tecnológica del Modelo Institucional Integral de Gestión Educativa Propuesto.

Elaboración propia (2019).

Hoy en día, la Dimensión Tecnológica está ligada a la innovación, por lo cual, es necesario que se fusionen los conceptos de tecnología e innovación organizacional, dando como resultado una valiosa combinación de crecimiento interno, en cada una de las problemáticas que en materia de tecnología se presenten en la institución educativa, siguiendo esta estrategia, no se podrá perder de vista que la tecnología es un elemento que regula el grado de modernidad y vanguardia que deba tener la organización.

Cada uno de los elementos de esta dimensión, han sido pensados para lograr integrar el grado de modernidad que pueda adaptar la institución educativa en cada uno de los rubros marcados en su estructura interna, cubriendo para ello, algunas características propias de la institución, de la mano con las necesidades y expectativas de los alumnos, personal académico y personal administrativo que formen parte de la institución educativa.

Se trata de una solución integral, que mantendrá un equilibrio entre cada uno de los rubros que conforman esta dimensión, que estará soportando soluciones de tecnología de vanguardia.

Normativa. Como parte de una estrategia transversal, que sirva como punto de referencia para velar por las buenas prácticas, de los procesos internos de las instituciones educativas, que adopten el Modelo Institucional Integral de Gestión Educativa, se propone la Dimensión Normativa, la cual servirá como referente normativo en cada una de las actividades que se desarrollen.

Básicamente esta Dimensión estará aportando los fundamentos jurídicos, que dan soporte y sustento legal a cada una de las acciones que se implementen a favor de la administración de los procesos internos.

Una de las problemáticas que se detectaron al momento de adoptar la presente propuesta de modelo de gestión educativa, es precisamente lo relacionado al cumplimiento de reglamentos, políticas y lineamientos institucionales.

Lo anterior ha ocasionado históricamente, algunos desacuerdos en cada una de las actividades a desempeñar.

\begin{tabular}{|l|l|}
\hline \multicolumn{1}{|c|}{ Elemento } & \multicolumn{1}{|c|}{ Descripción } \\
\hline Reglamentos & Reglamentos \\
Institucionales & institucionales y \\
& visibles en repositorios \\
& de acceso público. \\
\hline Manuales & Descripción de \\
organizacionales & manuales \\
\hline
\end{tabular}




\begin{tabular}{|c|c|}
\hline & $\begin{array}{l}\text { organizacionales con } \\
\text { las funciones de } \\
\text { manera específica. }\end{array}$ \\
\hline $\begin{array}{ll}\text { Contratos } & \text { con } \\
\text { proveedores } & \end{array}$ & $\begin{array}{lr}\text { Especificación } & \text { de } \\
\text { contratos } & \text { con } \\
\text { proveedores visibles } \\
\text { en repositorios de } \\
\text { acceso público. }\end{array}$ \\
\hline Licitaciones públicas & $\begin{array}{l}\text { Difusión de licitaciones } \\
\text { públicas para } \\
\text { incorporación de } \\
\text { nuevos proveedores. }\end{array}$ \\
\hline $\begin{array}{l}\text { Respaldo jurídico } \\
\text { externo }\end{array}$ & $\begin{array}{l}\text { Presencia permanente } \\
\text { de entidades de } \\
\text { carácter jurídico } \\
\text { institucional. }\end{array}$ \\
\hline
\end{tabular}

Tabla No. 5: Elementos de la Dimensión Normativa del Modelo Institucional Integral de Gestión Educativa Propuesto.

Elaboración propia (2019).

Por lo que resulta muy necesaria la incorporación de esta dimensión como parte del fundamento que estará regulando la articulación de las dimensiones que forman el modelo.

Operativa. La Dimensión operativa, es una forma de garantizar la sana funcionalidad interna de los elementos que de manera conjunta hacen posible la operación institucional.

En ese sentido se hace necesaria su incorporación con miras a lograr el descubrimiento e impulso de cada una de las potencialidades de los procesos internos de la institución educativa, básicamente se pretende mantener en armonía la operación cotidiana de la institución educativa, para así poder lograr un punto de equilibrio entre los procesos internos de la institución, llámese, procesos administrativos, académicos y de gestión.

La dimensión operativa contiene elementos muy importantes que están vigentes y que forman parte del crecimiento organizacional.

\begin{tabular}{|ll|lr|}
\hline \multicolumn{2}{|c|}{ Elemento } & \multicolumn{2}{c|}{ Descripción } \\
\hline Manuales & de & Elaboración & y \\
Operación & & actualización & \\
& & permanente & de \\
& & manuales & de \\
\hline
\end{tabular}

\begin{tabular}{|c|c|}
\hline & operación. \\
\hline $\begin{array}{ll}\text { Documentación } & \text { de } \\
\text { sistemas } & \text { de } \\
\text { información } & \end{array}$ & $\begin{array}{l}\text { Sistemas de } \\
\text { Información } \\
\text { respaldados por } \\
\text { manuales de usuario y } \\
\text { manuales técnicos. }\end{array}$ \\
\hline $\begin{array}{l}\text { Bitácoras de control de } \\
\text { acceso de usuarios }\end{array}$ & $\begin{array}{l}\text { Generación de } \\
\text { bitácoras de control de } \\
\text { alumnos, profesores, } \\
\text { administrativos } \\
\text { invitados a las } \\
\text { instalaciones de la } \\
\text { institución educativa } \\
\text { que adopte el modelo. }\end{array}$ \\
\hline $\begin{array}{ll}\text { Mecanismos } & \text { de } \\
\text { seguridad en las } \\
\text { instalaciones }\end{array}$ & $\begin{array}{ll}\text { Establecimiento de } \\
\text { mecanismos de } \\
\text { seguridad dentro de } \\
\text { las instituciones } \\
\text { educativas. }\end{array}$ \\
\hline $\begin{array}{l}\text { Mantenimiento } \\
\text { permanente de las } \\
\text { instalaciones físicas }\end{array}$ & $\begin{array}{l}\text { Generación de un plan } \\
\text { de mantenimiento para } \\
\text { instalaciones físicas. }\end{array}$ \\
\hline
\end{tabular}

Tabla No. 6: Elementos de la Dimensión Operativa del Modelo Institucional Integral de Gestión Educativa Propuesto.

Elaboración propia (2019).

A través del paso del tiempo, esta dimensión operativa, estará retomando impulso y fuerza interna, con la finalidad de tener influencia en las prácticas laborales, que de manera cotidiana no hayan tenido éxito.

Es importante establecer dentro de esta dimensión, la importancia de llevar de manera permanente sesiones de capacitación para cada uno de los colaboradores de la institución educativa, respetando el rol con el cual participen dentro del equipo de trabajo, con la finalidad que desde su perspectiva, se encuentre claridad en el logro de los objetivos organizacionales que se planteen.

El objetivo de establecer una capacitación permanente en cada una de las áreas que conforman la institución educativa, dará la opción de mantener una actualización constante en temáticas acordes al nivel de conocimiento que se requiera en la ejecución de sus funciones y actividades propias del papel que desempeñen en la organización.

A nivel institución educativa, esto permitirá tener un nivel de prestigio mayor en el mercado, lo cual impactará en 
gran medida en el desarrollo de las funciones sustantivas que realice, siendo la académica una de las más importantes que permitirá reflejar el crecimiento institucional permanente.

Institucional. Un rasgo distintivo de cada institución educativa es el conjunto de reglas que forman el marco institucional a seguir, de acuerdo a Beltrán LLavador (2000), la dimensión institucional comprende un sistema de reglas que introduce un orden en el propio conjunto social de referencia en el que se construyen las organizaciones educativas, traduciendo en el interior de las mismas el orden social existente.

La anterior afirmación no se genera en el seno de las instituciones educativas, sino en la sociedad (obligatoriedad en la asistencia, tipos de agrupamientos homogeneizados, calendario escolar, distribución de los horarios), siendo las practicas organizativas un reflejo de las prácticas sociales a través de unos sistemas de significados que proporcionan percepciones compartidas inducidas por la institución mediante la acción persistente de la normativa.

Las instituciones educativas modernas, son el reflejo de la sana estructuración de esfuerzos para obtener objetivos comunes, donde se indique un verdadero espíritu de unión entre las personas que colaboran y reparten funciones a favor del cumplimiento de las metas organizacionales.

\begin{tabular}{|l|l|}
\hline \multicolumn{1}{|c|}{ Elemento } & \multicolumn{1}{|c|}{ Descripción } \\
\hline Politicas Educativas & $\begin{array}{l}\text { Permitirán la la } \\
\text { fortalecimiento de laciva, con } \\
\text { calidad educativa } \\
\text { la finalidad de obtener } \\
\text { buenos resultados en } \\
\text { la cobertura de los } \\
\text { servicios que ofrezca } \\
\text { la institución } \\
\text { educativa. }\end{array}$ \\
\hline Calidad Educativa & $\begin{array}{l}\text { Como una forma de } \\
\text { promover un procesos } \\
\text { de mejora continua en } \\
\text { cada uno de los } \\
\text { procedimientos } \\
\text { administrativos. }\end{array}$ \\
\hline Imagen Institucional & $\begin{array}{l}\text { Permitirá aumentar el } \\
\text { nivel de desarrollo del } \\
\text { espíritu de pertenencia }\end{array}$ \\
\hline
\end{tabular}

\begin{tabular}{|c|c|}
\hline & $\begin{array}{l}\text { a la institución } \\
\text { educativa que adopte } \\
\text { el modelo propuesto. }\end{array}$ \\
\hline Prácticas organizadas & $\begin{array}{l}\text { Establecimiento de } \\
\text { mecanismos de control } \\
\text { en cada una de las } \\
\text { prácticas } \\
\text { administrativas que se } \\
\text { lleven a cabo al interior } \\
\text { de la organización } \\
\text { educativa. }\end{array}$ \\
\hline $\begin{array}{l}\text { Presencia de un } \\
\text { modelo educativo }\end{array}$ & $\begin{array}{l}\text { Como respaldo básico } \\
\text { de la estructura } \\
\text { organizacional que se } \\
\text { mantenga en la } \\
\text { institución educativa } \\
\text { que adopte el modelo } \\
\text { propuesto. }\end{array}$ \\
\hline
\end{tabular}

Tabla No. 7: Elementos de la Dimensión Institucional del Modelo Institucional Integral de Gestión Educativa Propuesto.

Elaboración propia (2019).

De acuerdo a Gento y Montes (2010), en su artículo "Como Elaborar un Diagnóstico de la Calidad de un Centro Educativo: Implementación y Resultados de un Modelo", se menciona que:

Si la calidad de la educación es hoy una necesidad generalmente sentida por los individuos y los grupos humanos para su propio desarrollo y progreso, ningún sistema educativo puede considerar totalmente lograda dicha calidad, si no incluye el adecuado tratamiento educativo, acomodado a dicho paradigma de calidad, a todas las personas, incluidas aquellas que presentan alguna especial necesidad o diversidad.

\section{Discusión}

Durante el contacto que se tuvo con instituciones educativas de nivel superior, en el proceso de recopilación de información, fue posible identificar la complejidad dentro de la demanda de obtención de procesos flexibles en su organización interna, los principales hallazgos detectados son los siguientes:

- Se encontró que las instituciones educativas encuestadas cuentan con la posibilidad de 
inversión en la integración de modelos de gestión educativa que permitan ofrecer soluciones a las necesidades de información, ante la demanda de procesos administrativos sólidos, sin embargo, considerando a las finanzas como un tema delicado, es necesario realizar una labor de justificación importante que permita convencer a las autoridades respectivas para tomar la decisión de invertir en la mejora de procesos organizacionales.

- Se mantiene una preocupación latente en cada una de las figuras presentes en las instituciones educativas de nivel superior encuestadas, con la visión de lograr una estabilidad organizacional digna de un crecimiento sólido.

\section{Conclusiones}

- Con la implementación del modelo institucional integral de gestión educativa, se promueve un mejoramiento organizacional en donde la participación activa de directivos, profesores y alumnos, fomentará la adquisición de beneficios internos que serán reflejados en su actuar cotidiana, desde ámbitos administrativos y académicos.

- A través de la implementación del modelo institucional integral de gestión educativa se estarán fortaleciendo el diseño de proyectos a favor de la institución que lo adopte, adquiriendo control institucional y la posibilidad de administrar recursos, con el enfoque de proyectos que fomenten el ahorro institucional.

- Se estará promoviendo el liderazgo dentro de la institución que adopte el modelo, factor clave de crecimiento organizacional, en la generación de buenas prácticas asociadas al fortalecimiento del capital humano.

- Se generará una sana convivencia dentro de la institución, con la firme convicción de integrarse a las diversas figuras inmersas en el desarrollo de funciones sustantivas que impacten el desarrollo y consolidación integral, factor clave para lograr la consolidación institucional.

- Se logra establecer mecanismos de posicionamiento en el mercado, a través de un modelo de gestión educativa sólido, que contempla de manera integral los principales elementos que la institución educativa requiere para lograr su consolidación a corto plazo.

\section{Referencias}

1. SEP Secretaría de Educación Pública (2018). La estructura del Sistema Educativo Mexicano. (2a ed.). Ariel. Obtenido de http://www.sep.gob.mx/work/models/sep1/Resource/1447/1/images/ sistemaedumex09_01.pdf

2. Hernández Sampieri, R. (2007). Fundamentos de Metodología de la Investigación. McGraw-Hill, Madrid, España.

3. Flores Briseño, M. (2012). Gestión Institucional en Educación a Distancia. UDG Virtual: Universidad de Guadalajara, México.

4. Barreda, H. (2007). Características distintivas en la gestión del servicio educativo. Gestao Universitaria na América Latina-GUAL, 1 (1). Recuperado de https://periodicos.ufsc.br/index.php/gual/article/view/24996

5. Instituto Internacional de Planeamiento de la Educación (2001). Gestión Educativa Estratégica, diez módulos destinados a los responsables de los procesos de transformación educativa: https://educrea.cl/gestion-educativa-estrategica-diez-modulosdestinados-a-los-responsables-de-los-procesos-de-transformacioneducativa/

6. Correa, A. (2009), Fundación Universitaria Luis Amigó, Colombia. La Gestión Educativa, un nuevo paradigma. http://virtual.funlam.edu.co/repositorio/sites/default/files/6lagestione ducativaunnuevoparadigma.pdf

7. Programa Escuelas de Calidad, Secretaría de Educación Pública (2010):

http://formacion.sigeyucatan.gob.mx/formacion/materiales/5/d3/p3/3 $\% 20$ EL $\% 20$ MODELO $\% 20$ DE $\% 20$ GESTION\%20EDUCATIVA $\% 2$ OESTRATEGICA.pdf

8. Morone, G., (26 de diciembre de 2018), Métodos y técnicas de la investigación científica. Obtenido de http://biblioteca.ucv.cl/site/servicios/documentos/metodologias_inve stigacion.pdf 